\title{
Cyanide-Bridged $\mathrm{W}^{\mathrm{V}}$-Co" Double Zigzag Chain Based on an Octacoordinated W Precursor: Metamagnetism and Spin Canting
}

Jung Hee Yoon, ${ }^{\dagger}$ Jeong Hak Lim, ${ }^{\dagger}$ Seok Woo Choi, ${ }^{\dagger}$ Hyoung Chan Kim,,${ }^{\dagger}$ and Chang Seop Hong*,

Department of Chemistry and Center for Electro- and Photo-Responsive Molecules, Korea University, Seoul 136-701, Korea, and Systems Research Team, Research \& Development Division, Nuclear Fusion Research Center, Daejeon 305-333, Korea 


\section{Preparation:}

$\left(\mathrm{AsPh}_{4}\right)\left[\mathrm{W}(\mathrm{CN})_{6}(\mathrm{bpy})\right]^{1}(0.24 \mathrm{mmol})$ in $\mathrm{MeCN}$ was slowly added to $\mathrm{Co}\left(\mathrm{ClO}_{4}\right)_{2} \cdot 6 \mathrm{H}_{2} \mathrm{O}$ $(0.12 \mathrm{mmol})$ in $\mathrm{H}_{2} \mathrm{O}$. The resulting red solution was stirred for $1 \mathrm{~h}$, and then filtered, left undisturbed in the dark. After several days the filtrate gave red crystals of $\mathbf{1}$ in a yield of $38 \%$. Elemental analysis (\%) calcd for $\mathrm{C}_{32} \mathrm{H}_{35} \mathrm{CoN}_{16} \mathrm{O}_{9.5} \mathrm{~W}_{2}: \mathrm{C} 31.4, \mathrm{H} 2.89, \mathrm{~N} 18.3$; Found: C 31.4, H 2.50, N 18.0.

1. Szklarzewicz, J. Inorg. Chim. Acta 1993, 205, 85.

Crystal data for $1: \mathrm{C}_{16} \mathrm{H}_{14} \mathrm{Co}_{0.5} \mathrm{~N}_{8} \mathrm{O}_{3} \mathrm{~W}, \mathrm{FW}=579.67$, triclinic, space group $\mathrm{P}-1$, $\mathrm{a}=$ 8.3504(6), $b=10.5157(8), c=11.8710(9) \AA, \alpha=99.5090(10), \beta=100.8330(10), \gamma=$ 107.0080(10) ${ }^{\mathrm{o}}, \mathrm{V}=951.76(12) \AA^{3}, \mathrm{Z}=2, \rho_{\text {calcd }}=2.023 \mathrm{~g} \mathrm{~cm}^{-3}, \mu=6.525 \mathrm{~mm}^{-1}, 9999$ reflections collected, 3905 unique $\left(\mathrm{R}_{\text {int }}=0.0141\right), \mathrm{R} 1=0.0140$, wR2 $=0.0315$ [I > $2 \sigma(\mathrm{I})]$.

Table S1. Hydrogen bonds.

\begin{tabular}{lccccl}
\hline D-H & $\mathrm{d}(\mathrm{D}-\mathrm{H})$ & $\mathrm{d}(\mathrm{H} . . \mathrm{A})$ & $<\mathrm{DHA}$ & $\mathrm{d}(\mathrm{D} . . \mathrm{A})$ & $\mathrm{A}[$ Symmetry code $]$ \\
\hline O1-H1A & $0.74(4)$ & $2.26(4)$ & $163(4)$ & $2.972(4)$ & $\mathrm{N} 3[-\mathrm{x}+1,-\mathrm{y}+2,-\mathrm{z}+2]$ \\
O1-H1B & $0.84(4)$ & $1.81(4)$ & $175(4)$ & $2.645(4)$ & $\mathrm{O} 3[\mathrm{x}+1, \mathrm{y}+1, \mathrm{z}]$ \\
$\mathrm{O} 2-\mathrm{H} 2 \mathrm{~A}$ & $1.16(10)$ & $1.60(10)$ & $172(10)$ & $2.751(4)$ & $\mathrm{N} 2[-\mathrm{x}+1,-\mathrm{y}+1,-\mathrm{z}+1]$ \\
O2-H2B & $1.18(9)$ & $1.85(8)$ & $153(6)$ & $2.941(4)$ & $\mathrm{N} 5[-\mathrm{x}+1,-\mathrm{y}+2,-\mathrm{z}+1]$ \\
O2-H2B & $1.18(9)$ & $2.29(8)$ & $106(5)$ & $2.848(4)$ & $\mathrm{N} 3[-\mathrm{x}+1,-\mathrm{y}+2,-\mathrm{z}+2]$ \\
O3-H3A & $0.98(4)$ & $1.90(4)$ & $146(3)$ & $2.773(4)$ & $\mathrm{O} 2[-\mathrm{x}+1,-\mathrm{y}+1,-\mathrm{z}+1]$ \\
O3-H3B & $0.86(4)$ & $1.97(4)$ & $158(4)$ & $2.791(4)$ & $\mathrm{O} 2[\mathrm{x}, \mathrm{y}, \mathrm{z}+1]$ \\
\hline
\end{tabular}




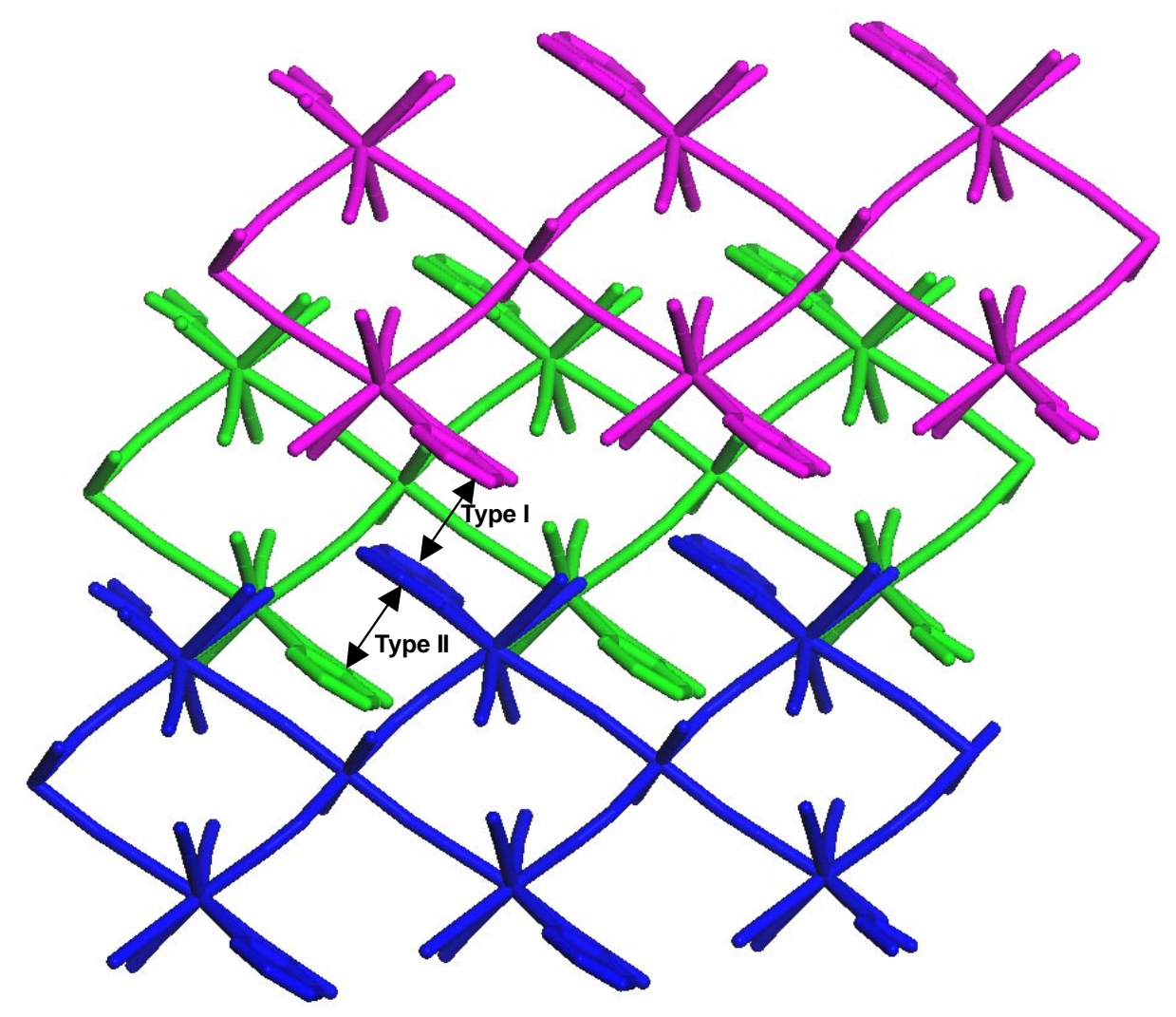

Figure S1. Extended structure of 1 showing $\pi-\pi$ interactions between pyridyl rings of bpy ligands. The supramolecular sheet formed by chains in pink and blue through type I $\pi-\pi$ interactions is connected with a neighboring chain indicated in green, finally leading to a three-dimensional network structure. 


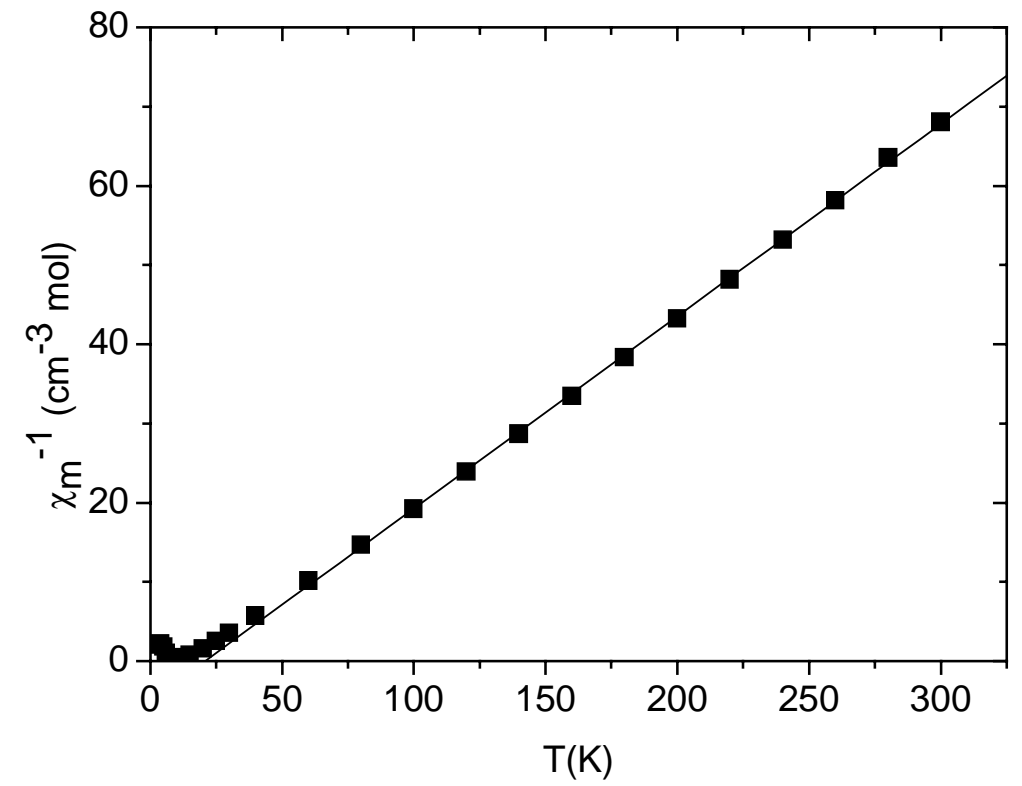

Figure S2. Plot of $\chi_{\mathrm{m}}{ }^{-1}$ versus T. The solid line represents the best fit of data with the Curie-Weiss equation. 

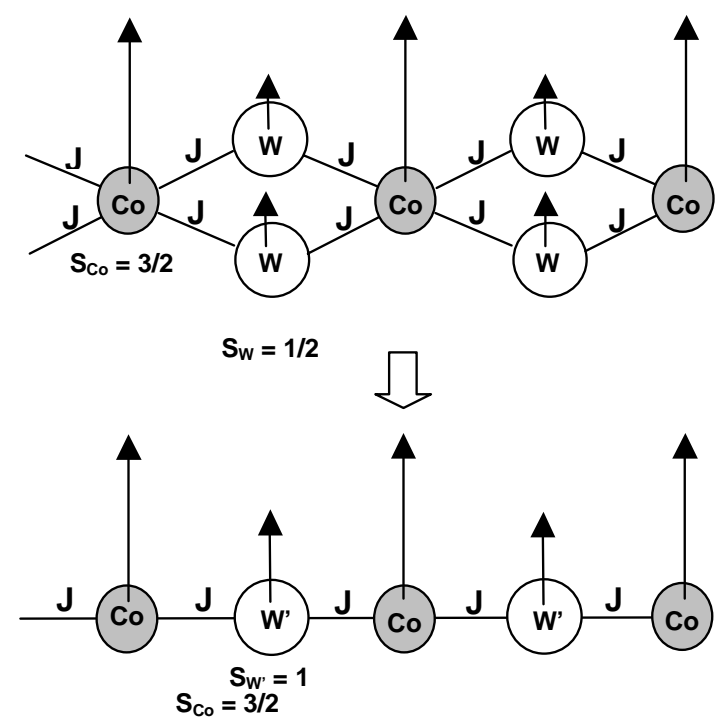

Figure S3. A possible magnetic model for exchange coupling between $\mathrm{W}^{\mathrm{V}}\left(\mathrm{S}_{\mathrm{W}}=1 / 2\right)$ and $\mathrm{Co}^{\mathrm{II}}\left(\mathrm{S}_{\mathrm{Co}}=3 / 2\right)$ spins in a chain.

Exact expression for the magnetic susceptibility is given below. ${ }^{1}$

$$
\begin{aligned}
\chi_{\mathrm{m}} \mathrm{T}=\left(\mathrm{N} \beta^{2} / 3 \mathrm{k}\right)\left[\mathrm{g}^{2}(1+\mathrm{u}) /(1-\mathrm{u})+\delta^{2}(1-\mathrm{u}) /(1+\mathrm{u})\right] \\
\mathrm{g}=\left(\mathrm{g}_{\mathrm{Co}}{ }^{\mathrm{e}}+\mathrm{g}_{\mathrm{W}},{ }^{\mathrm{e}}\right) / 2 \\
\delta=\left(\mathrm{g}_{\mathrm{Co}}{ }^{\mathrm{e}}-\mathrm{g}_{\mathrm{W}},{ }^{\mathrm{e}}\right) / 2 \\
\mathrm{u}=\operatorname{coth}\left(\mathrm{J}^{\mathrm{e}} / \mathrm{kT}\right)-\left(\mathrm{kT} / \mathrm{J}^{\mathrm{e}}\right)
\end{aligned}
$$

with $\mathrm{g}_{\mathrm{Co}}{ }^{\mathrm{e}}=\mathrm{g}_{\mathrm{Co}}\left[\mathrm{S}_{\mathrm{Co}}\left(\mathrm{S}_{\mathrm{Co}}+1\right)\right]^{1 / 2}, \quad \mathrm{~g}_{\mathrm{W}}{ }^{\mathrm{e}}=\mathrm{g}_{\mathrm{W}},\left[\mathrm{S}_{\mathrm{W}},\left(\mathrm{S}_{\mathrm{W}},+1\right)\right]^{1 / 2}$ and $\mathrm{J}^{\mathrm{e}}=$ $\mathrm{J}\left[\mathrm{S}_{\mathrm{Co}}\left(\mathrm{S}_{\mathrm{Co}}+1\right) \mathrm{S}_{\mathrm{W}},\left(\mathrm{S}_{\mathrm{W}},+1\right)\right]^{1 / 2}$.

1. Coronado, M. E.; Beltran, D.; Georges, R. Chem. Phys. 1983, 79, 449. 


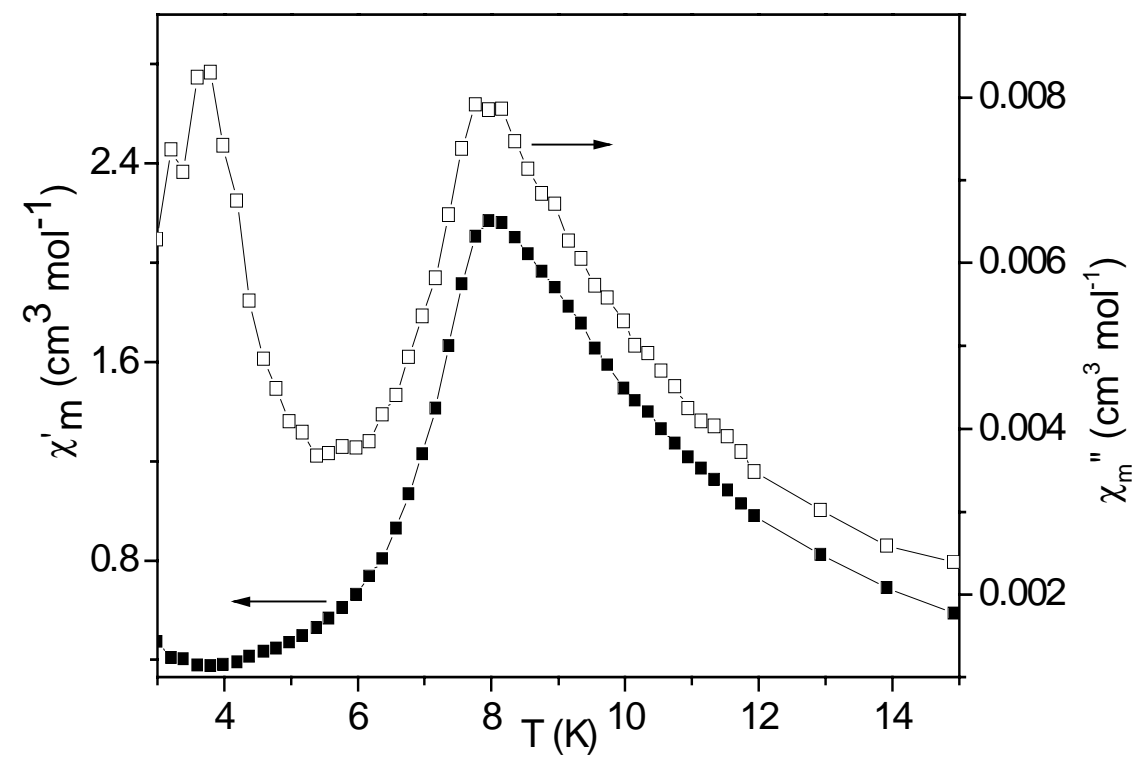

Figure S4. Plot of $\chi_{\mathrm{m}}$ ' (left) and $\chi_{\mathrm{m}}$ " (right) versus $\mathrm{T}$ at an ac field of $5 \mathrm{G}$, a frequency of $3000 \mathrm{~Hz}$, and zero dc field. 


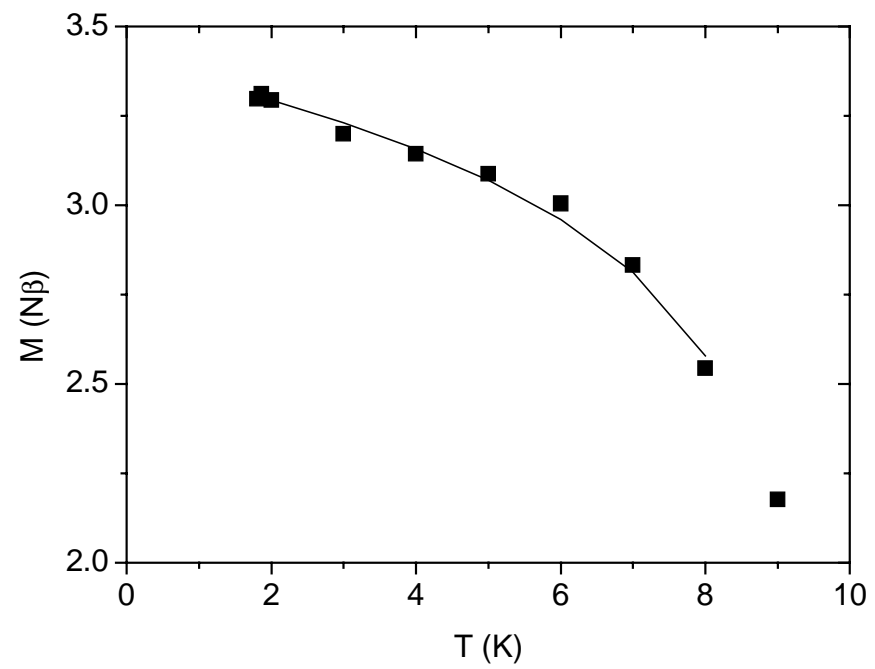

Figure S5. Plot of $M$ versus $T$. The solid line presents the best fit of data with the $M_{O}(1-$ $\left.\mathrm{T} / \mathrm{T}_{\mathrm{C}}\right)^{\beta}$ as $\mathrm{T} \rightarrow \mathrm{T}_{\mathrm{C}}$ from below, giving a critical exponent of $\beta=0.126$. 